\title{
Generalized net model of artificial bee colony optimization algorithm with intuitionistic fuzzy parameter adaptation
}

\author{
Dafina Zoteva $^{1,2}$, Olympia Roeva ${ }^{1}$ and Vassia Atanassova ${ }^{1}$ \\ ${ }^{1}$ Department of Bioinformatics and Mathematical Modelling \\ Institute of Biophysics and Biomedical Engineering, Bulgarian Academy of Sciences \\ e-mails: olympia@biomed.bas.bg, vassia.atanassova@gmail.com \\ ${ }^{2}$ Department of Computer Informatics, Faculty of Mathematics and Informatics \\ Sofia University "St. Kliment Ohridski" \\ e-mail: dafy.zoteva@gmail.com
}

Received: 10 February 2018

Accepted: 7 March 2018

\begin{abstract}
A Generalized Net (GN) model of Intuitionistic Fuzzy Logic (IFL) control and parameter adaptation of the Artificial Bee Colony (ABC) algorithm is proposed in the present paper. The developed GN-model describes the internal logic of the ABC algorithm with an embedded IFL controller to determine the magnitude of perturbation, depending on the current iteration of the algorithm.
\end{abstract}

Keywords: Generalized net, Intuitionistic fuzzy logic, Artificial Bee Colony, Parameter adaptation.

AMS Classification: 03E72, 68Q85, 62H30.

\section{Introduction}

In order to increase the performance of an optimization algorithm, especially a metaheuristic algorithm, it is necessary to provide adjustments of its parameters depending on the specific problem. The algorithm parameters could be a static set or could be varying values during an algorithm run. So called parameter control is one of the main challenges of the field of optimization computation.

Finding robust control parameters setting is not a trivial task, since their interaction with the algorithm performance is a complex relationship and the optimal ones are problem-dependent [16]. An optimal or a near-optimal set of control parameters for one metaheuristic algorithm does 
not generalize to all cases. This stresses the need of efficient techniques that help finding good parameter settings for a given problem, i.e. the need of good parameter tuning methods.

In the literature, these are studies on new metaheuristic algorithms using fuzzy logic for parameter adaptation, e.g. ant colony optimization [2], bee colony optimization [3, 11], gravitational search algorithm [31], genetic algorithm [16, 24], bat algorithm [22, 29], etc. A survey on nature-inspired optimization algorithms with fuzzy logic for dynamic parameter adaptation is presented in [32].

Some works deal with the application of Intuitionistic Fuzzy Logic (IFL) [4, 8, 10] for dynamic parameter adaptation of algorithms. In [27], the use of IFL has been investigated to control Genetic Algorithm parameters and in [22] - for parameter adaptation of the Bat Algorithm. Authors in [27] propose a Generalized Net (GN) model describing the IFL control of the crossover and the mutation probability in the genetic algorithm.

So far, GNs [5-7, 9] have been used as a tool for modelling various metaheuristics: genetic algorithms [25, 28, 30], ant colony optimization [12] and the firefly algorithm [26].

A detailed description of the logic of the basic ABC algorithm within a GN-model is presented in [34]. ABC optimization is one of the efficient population-based biological-inspired algorithms. There are three continuous optimization algorithms based on intelligent behaviour of honeybee swarm $[17,23]$. The published results indicate that ABC can efficiently be used for many optimization problems $[18,19,21]$.

The performance of the $\mathrm{ABC}$ algorithm is very good for multidimensional basic functions. However, it can be improved when dealing with complex optimization problems. A large part of the proposed improvements is related to the search equation of the $\mathrm{ABC}$ algorithm. Here are some of them.

Gbest-guided $\mathrm{ABC}$ algorithm, which incorporates the information of the global best solution in the local search, is described in [33]. An improved search equation based on the best solution of the previous iteration is introduced in [13]. A version of the algorithm where the onlookers are focused directly on the global best solution and update this global best solution one by one is presented in [20]. The performance of the basic ABC algorithm is enhanced with Gaussian search in the onlookers' phase, parameter adaptation strategy and fitness-based search for neighbourhood mechanism in the employed bees' phase in [14].

Two other improvements of the ABC algorithm are discussed in [1]. Two additional control parameters are used for improving the convergence rate: a modification rate to control the frequency of perturbation and a scaling factor to control the magnitude of perturbation. The values of second parameter can be changed during the search based on the ratio of the successful mutations.

A new self-adaptive perturbation for the basic ABC algorithm is proposed in [15]. The values of the control parameter $\phi_{i j}$ are changed according to the current iteration. They are calculated in a different way for the employed bees' phase and for the onlookers' phase.

So, the authors are motivated to include in the developed GN-model of ABC algorithm a description of an IFL controller for dynamic parameter adaptations of ABC parameter $\phi_{i j}$.

The main objective of this research is to propose an IFL controller embedded in the GNmodel for the purpose of calculating the magnitude of perturbation $\left(\phi_{i j}\right)$ for each iteration of the ABC algorithm. 


\section{Background of Artificial Bee Colony algorithm}

Artificial bee colony algorithm is a swarm algorithm introduced by Karaboga [17] that simulates the foraging behaviour of the honey bees. $\mathrm{ABC}$ is one of the competitive optimization algorithms that is highly effective for the purpose of multi-dimensional numerical problems.

The algorithm can be described roughly as follows.

The initialization phase of the algorithm is responsible for setting the initial values of the algorithm parameters: the population size $(N P)$, the number of the food sources $(S N)$, the number of parameters ( $D$, dimension of the search space), the maximum number of trials before abandoning a food source (limit) and the maximum number of iterations or cycles $(M C N)$.

The initial population of food sources is generated during the initialization phase. Equation (1) is used for generating each of the food sources:

$$
x_{i}^{j}=x_{\min }^{j}+\operatorname{rand}(0,1)\left(x_{\max }^{j}-x_{\min }^{j}\right),
$$

where $i \in[1 ; S N], j \in[1 ; D], x_{\min }^{j}$ and $x_{\text {max }}^{j}$ are the lower $(L b)$ and the upper $(U b)$ bounds of the dimension $j, S N$ is the count of the food sources. The number of trials for each of the generated food sources is set to 0 . This counter will be incremented each time the food source associated with it has not been replaced by a better one.

Each iteration of the algorithm includes the following three phases: an employed bees' phase, an onlookers' phase and a scouts' phase.

The employed bees search for new food sources around the one stored in their memory, based on the equation (2):

$$
v_{i j}=x_{i j}+\phi_{i j}\left(x_{i j}-x_{k j}\right)
$$

where $j$ is a random integer number in the range $[1 ; D], k$ is randomly selected index, $k \in[1 ; S N], k \neq i . \phi_{i j} \in[-1 ; 1]$ is a random number.

During the onlookers' phase the onlookers choose a food source depending on the probability value $p_{i}$ associated with that food source:

$$
p_{i}=\frac{f_{i}}{\sum_{n=1}^{S N} f_{n}}
$$

where $f_{i}$ is the fitness value of the solution $i$ and it is proportional to the nectar amount of the food source in position $i$.

The scouts' phase begins after the employed bees and the onlookers finish their search. The value of the trials for each food source is compared to the limit, set in the initialization phase. If the value of the trials exceeds the limit, the corresponding food source is abandoned. The abandoned food source is replaced by a food source generated randomly by equation (1), i.e. a food source found by a scout.

The algorithm stops when the predefined maximum number of iterations $(M C N)$ is reached. 


\section{Intuitionistic fuzzy parameter adaptation}

\subsection{Intuitionistic fuzzy sets}

Intuitionistic fuzzy sets (IFSs) have been introduced by Atanassov [4, 8, 10] as an extension and generalization of the notion of the Zadeh's fuzzy sets. IFS is a set whose elements have degrees of membership and non-membership.

Let $E$ is a fixed universe, $E \neq \emptyset$. Let A be a subset of $E$. The IFS $A^{*}$ is defined as follows:

$$
\begin{gathered}
A^{*}=\left\{\left\langle x, \mu_{A}(x), v_{A}(x)\right\rangle \mid x \in E\right\}, \text { where } \\
\mu_{A}, v_{A}: E \rightarrow[0 ; 1], 0 \leq \mu_{A}(x)+v_{A}(x) \leq 1 .
\end{gathered}
$$

where $\mu_{A}(x)$ is the degree of membership, $v_{A}(x)$ is the degree of non-membership of an element $\mathrm{x}$ to the set A. Let $\pi_{A}(x)=1-\mu_{A}(x)-v_{A}(x)$. The function $\pi_{A}(x)$ determines the degree of uncertainty.

\subsection{Intuitionistic fuzzy logic system}

The basic structure of an intuitionistic fuzzy logic system (IFLS) defined in terms of IFS is presented in Figure 1.

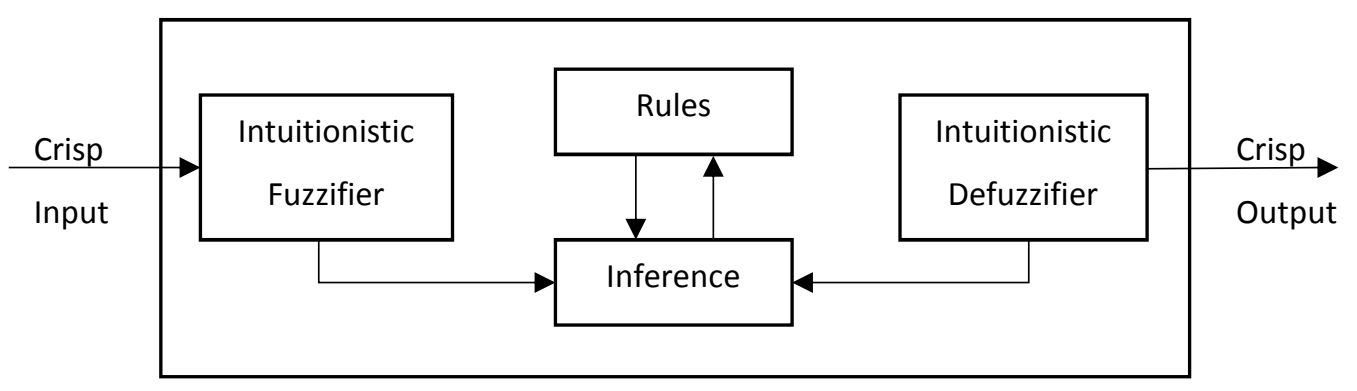

Figure 1. Basic structure of intuitionistic fuzzy logic system

The first step of the IFLS is to fuzzify the crisp value of the input parameter using its membership and non-membership functions. The intuitionistic fuzzy inference is based on IFTHEN rules. The actual task here is to compute the IF output sets derived by the IF input sets. The final step is to convert the IFS output of the rules into a crisp value.

The IFLS proposed in the present research uses Mamdani type inference method for the membership and non-membership functions.

The intuitionistic fuzzy logic controller (IFLC) takes a single input, the variable $I$ (Iteration), defined by the equation (4) in the range $[0 ; 1]$ :

$$
I=\frac{\text { current iteration }}{M C N}
$$

The membership and the non-membership functions of the input variable $I$ are presented in Figure 2. They are described by three IFS: Low, Middle and High. 


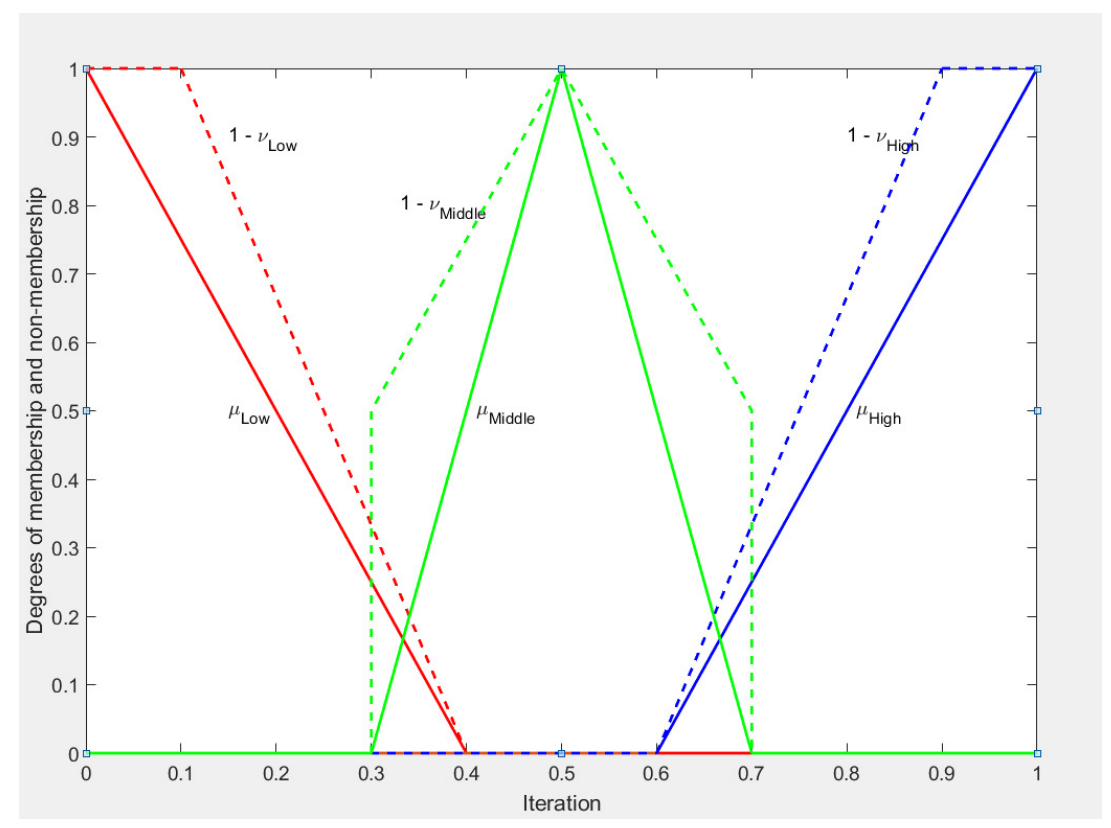

Figure 2. Membership and mon-membership functions for iteration

The output of the IFLC is the value of the magnitude of perturbation $\phi_{i j}$ used during the search of the employed bees and the onlookers, equation (2), where $\phi_{i j} \in[-1 ; 1]$.

The membership and the non-membership functions of the output parameter $\phi_{i j}$ are presented in Figure 3. They are also described by three IFS: Low, Middle and High.

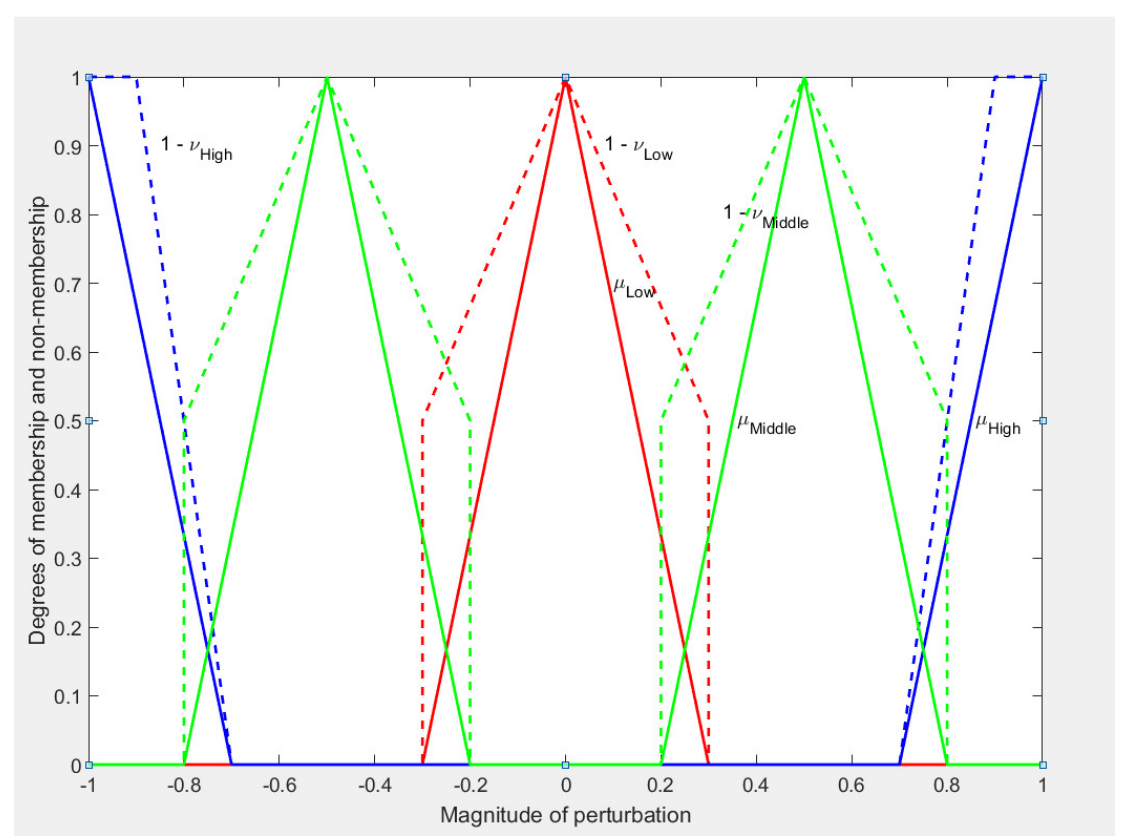

Figure 3. Membership and non-membership functions for $\phi_{i j}$

The rules of the system are three of Mamdani type, described as follows:

1) IF $I$ (Iteration) is Low THEN $\phi_{i j}$ is High.

2) IF $I$ (Iteration) is Middle THEN $\phi_{i j}$ is Middle.

3) IF $I$ (Iteration) is High THEN $\phi_{i j}$ is Low. 
When the iterations are Low, the value of the magnitude of perturbation is High. The perturbation from the original food source at this point is bigger. The aim is to explore larger areas around the current food source.

When the iterations are Middle, the value of the magnitude of perturbation is Middle.

When the iterations are High, the value of the magnitude of perturbation is Low. In this stage, at best the solutions have to converge to an optimal one. The perturbation should be kept smaller.

That way, a good balance between the exploration and the exploitation of the search could be found.

\section{Generalized net model of ABC optimization with IFL parameter adaptation}

Based on the GN-model of the ABC algorithm proposed in [34], the GN-model with IFL parameter adaptation is presented in Figure 4. Each of the main steps of the algorithm is associated with a transition of the developed GN-model.

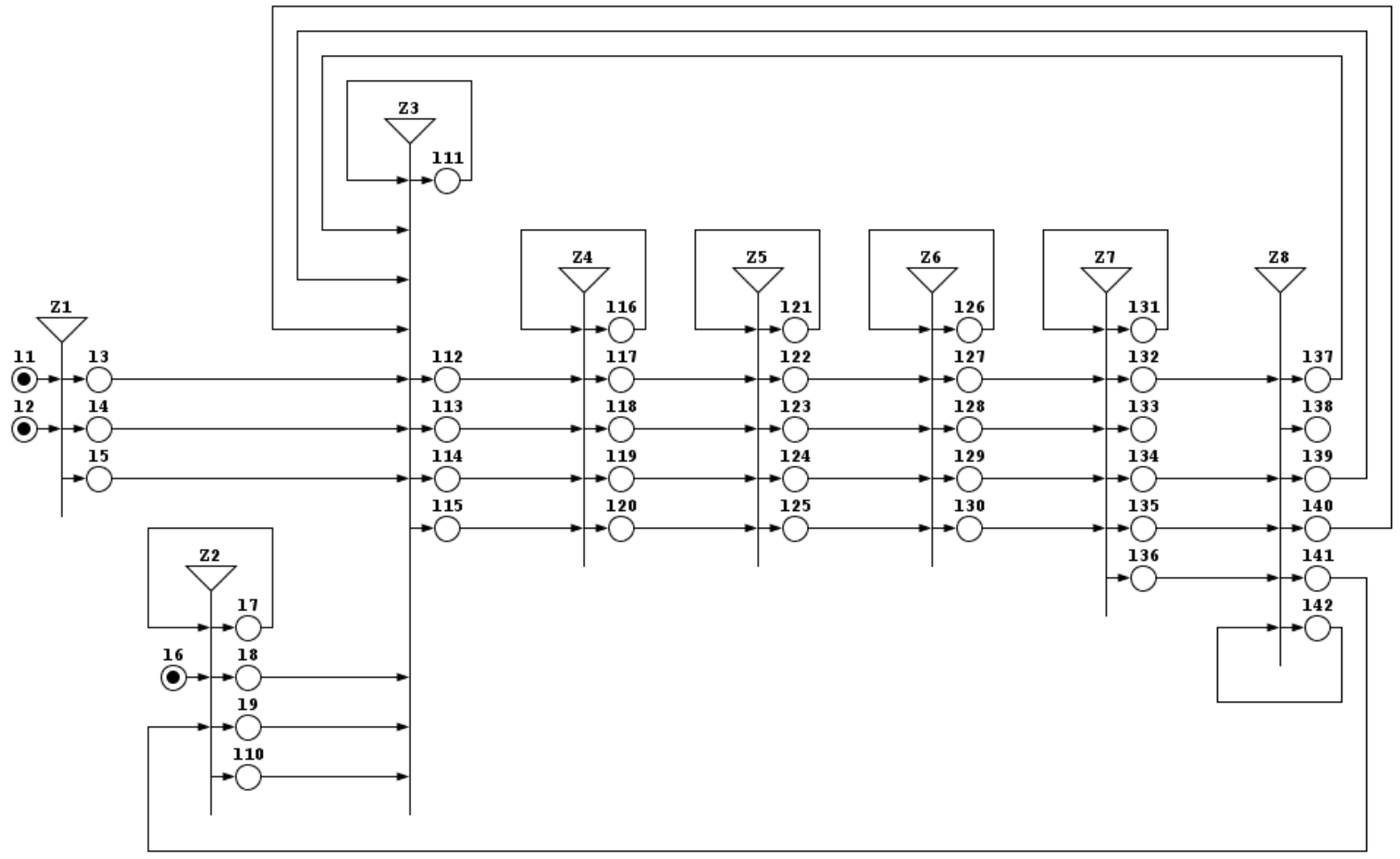

Figure 4. Generalized net model of ABC with IFL controller

The first transition $Z_{1}$ is responsible for the initialization step of the algorithm. The token $\chi$ enters the GN through the input place $l_{1}$ with initial characteristics " $A B C$ input parameters: $S N$, $D$, limit, $M C N$ ". The token $\delta$ enters the GN through the input place $1_{2}$ with initial characteristics "Problem parameters: objective function $f(x), L b$ and $U b$ ".

The form of the first transition $Z_{1}$ of the GN-model is the following: 


$r_{1}=$\begin{tabular}{c|ccc}
$Z_{1}=\left\langle\left\{l_{1}, l_{2}\right\}\right.$, & $\left.\left\{l_{3}, l_{4}, l_{5}\right\}, r_{1}, \wedge\left(l_{1}, l_{2}\right)\right\rangle$, \\
& $l_{3}$ & $l_{4}$ & $l_{5}$ \\
\hline$l_{1}$ & true & true & false \\
$l_{2}$ & true & false & true
\end{tabular}

The first transition can be activated only when both of the tokens $\chi$ and $\delta$ are present in their initial places. Each of the tokens $\chi$ and $\delta$ is split into two identical tokens upon activation. One copy of $\chi$ and one copy of $\delta$ merge into a new token $\alpha$ in the output place $l_{3}$. In place $l_{3}$, based on equation (1), the token $\alpha$ receives new characteristics "initial population $x_{i}, f\left(x_{i}\right)$ and trials $s_{i}=0$ ". At the same time, the other copies of the tokens $\chi$ and $\delta$ are transferred to output places $l_{4}$ and $l_{5}$, respectively.

The second transition $Z_{2}$ is related to the work of the embedded IFL controller that is responsible for the adaptation of the magnitude of perturbation $\phi_{i j}$. The token $\rho$ enters the GN through the input place $l_{6}$ with initial characteristic "current iteration $=0, M C N$ ".

The form of the second transition $Z_{2}$ is the following:

where

\begin{tabular}{r|cccc}
\multicolumn{4}{c}{$Z_{2}=\left\langle\left\{l_{6}, l_{7}, l_{41}\right\},\left\{l_{7}, l_{8}, l_{9}, l_{10}\right\}, r_{2}, \vee\left(l_{6}, l_{7}, l_{41}\right)\right\rangle}$, \\
$r_{2}=$ & $l_{7}$ & $l_{8}$ & $l_{9}$ & $l_{10}$ \\
\hline$l_{6}$ & true & false & false & false \\
$l_{7}$ & false & $W_{7,8}$ & $W_{7,9}$ & $W_{7,10}$ \\
$l_{41}$ & true & false & false & false
\end{tabular}

- $W_{7,8}=$ "max $\left(\mu_{\text {LowIter }}(\mathrm{I}), \mu_{\text {Miditer }}(\mathrm{I}), \mu_{\text {HighIter }}(\mathrm{I})\right)=\mu_{\text {LowIter }}(\mathrm{I}) "$,

- $W_{7,9}=" \max \left(\mu_{\text {LowIter }}(\mathrm{I}), \mu_{\text {MidIter }}(\mathrm{I}), \mu_{\text {HighIter }}(\mathrm{I})\right)=\mu_{\text {MidIter }}(\mathrm{I}) "$,

- $W_{7,10}=$ "max $\left(\mu_{\text {LowIter }}(\mathrm{I}), \mu_{\text {Miditer }}(\mathrm{I}), \mu_{\text {HighIter }}(\mathrm{I})\right)=\mu_{\text {HighIter }}(\mathrm{I}) "$.

The second transition $\mathrm{Z}_{2}$ can be activated when there is a token in one of the places $l_{6}, l_{7}$ or $l_{41}$. Each time the token $\rho$ is moved from an input place to place $l_{7}$ an additional 1 is added to the value of the initial characteristic "current iteration". When the token $\rho$ is moved from place $l_{7}$ to one of the output places $l_{8}, l_{9}$ or $l_{10}$ during the first iteration, it receives an additional characteristic " $\phi_{i j}$ ". After that first iteration, each time the token $\rho$ is transferred from place $l_{7}$ to one of the output places, the value of the characteristic " $\phi_{i j}$ " is changed. The new value of the magnitude of perturbation " $\phi_{i j}$ " is actually the value calculated by the IFL controller for this particular iteration of the algorithm.

The next two transitions are related to the phase of the employed bees.

The transition $Z_{3}$ is responsible for generating the new food sources neighbouring the ones, currently exploited by the employed bees. The form of the third transition is:

$$
\begin{aligned}
Z_{3}= & \left\langle\left\{l_{3}, l_{4}, l_{5}, l_{8}, l_{9}, l_{10}, l_{11}, l_{37}, l_{39}, l_{40}\right\},\left\{l_{11}, l_{12}, l_{13}, l_{14}, l_{15}\right\}, r_{3},\right. \\
& \left.\vee\left(\wedge\left(l_{3}, l_{4}, l_{5}, \vee\left(l_{8}, l_{9}, l_{10}\right)\right), l_{11}, \wedge\left(l_{37}, l_{39}, l_{40}, \vee\left(l_{8}, l_{9}, l_{10}\right)\right)\right)\right\rangle,
\end{aligned}
$$




\begin{tabular}{c|ccccc}
$r_{3}=$ & $l_{11}$ & $l_{12}$ & $l_{13}$ & $l_{14}$ & $l_{15}$ \\
\hline$l_{3}$ & true & false & false & false & false \\
$l_{4}$ & true & false & true & false & false \\
$l_{5}$ & true & false & false & true & false \\
$l_{8}$ & true & false & false & false & true \\
$l_{9}$ & true & false & false & false & true \\
$l_{10}$ & true & false & false & false & true \\
$l_{11}$ & false & true & false & false & false \\
$l_{37}$ & true & false & false & false & false \\
$l_{39}$ & true & false & true & false & false \\
$l_{40}$ & true & false & false & true & false
\end{tabular}

In order for the third transition to be activated for the first time, there should be tokens in $l_{3}$, $l_{4}, l_{5}$ and in one of the places $l_{8}, l_{9}$ or $l_{10}$, simultaneously. Later for the activation of the third transition, there should be a token in place $l_{11}$ or at the end of the current iteration, the tokens should be placed in $l_{37}, l_{39}, l_{40}$ and again in one of the places $l_{8}, l_{9}$ or $l_{10}$, simultaneously.

During the first iteration $\chi$ is stored in $l_{4}, \delta$ is stored in $l_{5}$ and $\rho$ is in one of the places $l_{8}, l_{9}$ or $l_{10}$. They split into two identical tokens each time they have to be transferred. Their copies $(\chi, \delta$ and $\rho$ ) are moved into places $l_{13}, l_{14}$ and $l_{15}$ respectively without receiving any additional characteristics.

The other copies of $\chi, \delta$ and $\rho$ along with the token $\alpha$ from place $l_{3}$ are merged into a new token $\beta$ in place $l_{11}$ during the first iteration. After the first iteration the copies of $\chi$ and $\delta$ are stored in places $l_{39}$ and $l_{40}$. They merge with the token from place $l_{37}$ and the token $\rho$ from one of the places $l_{8}, l_{9}$ or $l_{10}$ into a new token $\beta$ in place $l_{11}$. When this new token $\beta$ is moved to $l_{12}$, based on equation (2), it gains new characteristics "new solutions $v_{i j}$ and their corresponding evaluations $f_{i}$ ".

The fourth transition $Z_{4}$ is where the final part of the employed bees' phase takes place. This is where the greedy selection between the new generated food sources and the ones in the memories of the employed bees is applied. The form of transition $Z_{4}$ of the GN-model is the following:

$Z_{4}=\left\langle\left\{l_{12}, l_{13}, l_{14}, l_{15}, l_{16}\right\},\left\{l_{16}, l_{17}, l_{18}, l_{19}, l_{20}\right\}, r_{4}, \vee\left(\wedge\left(l_{12}, l_{13}, l_{14}, l_{15}\right), l_{16}\right)\right\rangle$,

The fourth transition $Z_{4}$ can be activated when there are tokens in the input places $l_{12}, l_{13}, l_{14}$ and $l_{15}$ simultaneously or there is a token in place $l_{16}$.

Copies of the tokens $\chi, \delta$ and $\rho$ are moved to $l_{18}, l_{19}$ and $l_{20}$, respectively. The token $\beta$ is merged with the other copies of the tokens $\chi, \delta$ and $\rho$ into a new token $\gamma$ in place $l_{16}$. The greedy selection is applied here between the new generated solutions and the corresponding old ones. When the 
token $\gamma$ is moved from place $l_{16}$ to place $l_{17}$, it receives additional characteristics "results of the greedy selection process, corresponding $f_{i}$, changed trials $s_{i}$ and calculated probabilities $p_{i j}$ ". The probability values will be used to select food sources during the onlookers' phase later.

The transitions $Z_{5}$ and $Z_{6}$ are related to the onlookers' phase. The fifth transition is responsible for generating and evaluating new food sources based on the roulette wheel selection. The form of the transition $Z_{5}$ of the GN-model is:

$Z_{5}=\left\langle\left\{l_{17}, l_{18}, l_{19}, l_{20}, l_{21}\right\},\left\{l_{21}, l_{22}, l_{23}, l_{24}, l_{25}\right\}, r_{5}, \vee\left(\wedge\left(l_{17}, l_{18}, l_{19}, l_{20}\right), l_{21}\right)\right\rangle$,

The fifth transition $Z_{5}$ can be activated when there are tokens in the input places $l_{17}, l_{18}, l_{19}$ and $l_{20}$ simultaneously or there is a token in place $l_{21}$.

Copies of the tokens $\chi, \delta$ and $\rho$ are moved to $l_{23}, l_{24}$ and $l_{25}$, respectively. The token $\gamma$ is merged with the other copies of the tokens $\chi, \delta$ and $\rho$ into a new token $\varepsilon$ in place $l_{21}$. In place $l_{21}$ the token $\varepsilon$ receives a new characteristic "evaluated new solutions for the onlookers from the solutions $x_{i j}$ selected depending on $p_{i j}$ ", based on equation (2). When the token $\varepsilon$ is transferred from place $l_{21}$ to place $l_{22}$, the additional characteristics "new solutions for the onlookers and their corresponding $f_{i}$ " are given to the token.

The transition $Z_{6}$ is related to the greedy selection process of the onlookers' phase. It has the following form:

$Z_{6}=\left\langle\left\{l_{22}, l_{23}, l_{24}, l_{25}, l_{26}\right\},\left\{l_{26}, l_{27}, l_{28}, l_{29}, l_{30}\right\}, r_{6}, \vee\left(\wedge\left(l_{22}, l_{23}, l_{24}, l_{25}\right), l_{26}\right)\right\rangle$,

The sixth transition $Z_{6}$ can be activated when there are tokens in the input places $l_{22}, l_{23}, l_{24}$ and $l_{25}$ simultaneously or there is a token in place $l_{26}$.

Copies of the tokens $\chi, \delta$ and $\rho$ are moved to $l_{28}, l_{29}$ and $l_{30}$, respectively. The token $\varepsilon$ is merged with the other copies of the tokens $\chi, \delta$ and $\rho$ into a new token $\zeta$ in place $l_{26}$. The greedy selection during the onlookers' phase is applied between the new generated solutions and the corresponding old ones upon entering place $l_{26}$. When the token $\zeta$ is transferred from place $l_{26}$ to place $l_{27}$, the "results of the greedy selection process for the onlookers, corresponding $f_{i}$, changed trials ${ }_{i}$ " are added to the list of characteristics of the token $\zeta$. 
The transition $Z_{7}$ describes the scout bee phase, where the food sources that would be abandoned will be identified. It has the following form:

\begin{tabular}{r|cccccc}
$Z_{7}=\left\langle\left\{l_{27}, l_{28}, l_{29}\right.\right.$, & $\left.l_{30}, l_{31}\right\}$, & \multicolumn{6}{c}{$l_{31}, l_{32}, l_{33}}$, & $\left.\left.l_{34}, l_{35}, l_{36}\right\}, r_{7}, \vee\left(\wedge\left(l_{27}, l_{28}, l_{29}, l_{30}\right), l_{31}\right)\right\rangle$, \\
$r_{7}=$ & $l_{31}$ & $l_{32}$ & $l_{33}$ & $l_{34}$ & $l_{35}$ & $l_{36}$ \\
\hline$l_{27}$ & true & false & false & false & false & false \\
$l_{28}$ & true & false & false & true & false & false \\
$l_{29}$ & true & false & false & false & true & false \\
$l_{30}$ & true & false & false & false & false & true \\
$l_{31}$ & false & true & true & false & false & false
\end{tabular}

The seventh transition $Z_{7}$ can be activated when there are tokens in the input places $l_{27}, l_{28}, l_{29}$ and $l_{30}$ simultaneously or there is a token in place $l_{31}$.

Copies of the tokens $\chi, \delta$ and $\rho$ are moved to $l_{34}, l_{35}$ and $l_{36}$, respectively. The token $\zeta$ is merged with the other copies of the tokens $\chi, \delta$ and $\rho$ into a new token $\eta$ in place $l_{31}$. Here upon entering place $l_{31}$, the solutions that have not been improved after a certain number of attempts and thus have to be abandoned are determined.

The token $\eta$ is split into two tokens: one with the abandoned solutions and the second with the rest of the solutions that are to be explored further. When the token $\eta$ enters place $l_{32}$, the list of solutions is extended with random solutions, generated based on equation (1). These random solutions are to replace the abandoned ones. The additional characteristics "new solutions, corresponding $f_{i}$ and trials $s_{i}=0$ " is added to the characteristics of the token $\eta$.

When the token $\eta$ is transferred from place $l_{31}$ to place $l_{33}$, the "abandoned solutions" are stored as a characteristic of the token.

The last transition $Z_{8}$ of the GN-model is responsible for selecting the best solution during the current iteration. All the remain solutions are evaluated. The transition $Z_{8}$ has the following form:

$Z_{8}=\left\langle\left\{l_{32}, l_{34}, l_{35}, l_{36}, l_{42}\right\},\left\{l_{37}, l_{38}, l_{39}, l_{40}, l_{41}, l_{42}\right\}, r_{8}, \vee\left(\wedge\left(l_{32}, l_{34}, l_{35}, l_{36}\right), l_{42}\right)\right\rangle$,

where

- $W_{42,37}=$ "current iteration $<M C N$ ",

- $W_{42,38}=\neg W_{42,37}$.

The last transition $Z_{8}$ can be activated when there are tokens in the input places $l_{32}, l_{34}, l_{35}$ and $l_{36}$ simultaneously or there is a token in place $l_{42}$.

Copies of the tokens $\chi, \delta$ and $\rho$ are moved to $l_{39}, l_{40}$ and $l_{41}$, respectively. The token $\eta$ is merged with the other copies of the tokens $\chi, \delta$ and $\rho$ into a new token $\theta$ in place $l_{42}$. In place $l_{42}$ the token $\theta$ receives new characteristics "ranked solutions, calculated corresponding $f_{i}$, the best solution of the current iteration and its corresponding $f_{\text {best" }}$. 
The token $\theta$ is transferred to place $l_{37}$ if the end of the $\mathrm{ABC}$ algorithm is not reached, i.e. the predicate $W_{42,37}$ is evaluated as true. If the end of the algorithm is reached, i.e. the predicate $W_{42,38}$ is evaluated as true, the token $\theta$ is transferred to place $l_{38}$ where "the best solution and its corresponding evaluation" are added as a final characteristic of the token.

\section{Conclusion}

In this paper the apparatus of generalized nets is used to describe the parameter adaptation of artificial bee colony optimization algorithm. The GN-model of ABC algorithm consists of 8 transitions and 42 places and performs the algorithm's basic procedures. An IFL controller is embedded in the GN-model for the purpose of calculating the magnitude of perturbation $\left(\phi_{i j}\right)$ for each iteration of the ABC algorithm. The application of the IFL controller can be extended to any other algorithm parameter or parameters as long as the predicates of the corresponding transition and the characteristic functions of the output places are changed.

\section{Acknowledgements}

Work presented here is partially supported by the National Scientific Fund of Bulgaria under the Grant DN02/10 “New Instruments for Knowledge Discovery from Data, and their Modelling”.

\section{References}

[1] Akay, B., \& Karaboga, D. (2012) A modified artificial bee colony algorithm for realparameter optimization. Information Sciences, 192, 120-142.

[2] Amador-Angulo, L. \& Castillo, O. (2016) A new fuzzy bee colony optimization with dynamic adaptation of parameters using interval type-2 fuzzy logic for tuning fuzzy controllers. Soft Computing, 1-24.

[3] Amador-Angulo, L. \& Castillo, O. (2015) Statistical analysis of type-1 and interval type-2 fuzzy logic in dynamic parameter adaptation of the BCO. Proceedings of the 2015 Conference of the International Fuzzy Systems Association and the European Society for Fuzzy Logic and Technology, 776-783.

[4] Atanassov, K. (1986) Intuitionistic fuzzy sets. Fuzzy Set and Systems, 20(1), 87-96.

[5] Atanassov, K. (1991) Generalized Nets. World Scientific, Singapore.

[6] Atanassov, K. (1997) Generalized Nets and Systems Theory. Academic Publishing House "Prof. M. Drinov", Sofia.

[7] Atanassov, K. (1998) Generalized Nets in Artificial Intelligence. Volume 1: Generalized Nets and Expert Systems. Academic Publishing House "Prof. M. Drinov", Sofia.

[8] Atanassov, K. (1999) Intuitionistic Fuzzy Sets: Theory and Applications, Springer, Heidelberg. 
[9] Atanassov, K. (2007) On Generalized Nets Theory. "Prof. Marin Drinov" Acad. Publ. House, Sofia.

[10] Atanassov, K. (2012) On Intuitionistic Fuzzy Sets Theory, Springer, Berlin.

[11] Caraveo, C., Valdez, F., \& Castillo, O. (2012) Optimization of fuzzy controller design using a new bee colony algorithm with fuzzy dynamic parameter adaptation. Applied Soft Computing, 43, 131-142.

[12] Fidanova, S., Atanassov, K., \& Marinov, P. (2011) Generalized Nets in Artificial Intelligence. Volume 5: Generalized Nets and Ant Colony Optimization. Prof. Marin Drinov Academyc Publishing House, Sofia.

[13] Gao, W.-F., \& Liu, S.-Y. (2012) A modified artificial bee colony algorithm. Computers \& Operations Research, 39, 687-697.

[14] Gao, W.-F., Chan, T. S. F., Huang, L., \& Liu, S. (2015) Bare bones artificial bee colony algorithm with parameter adaptation and fitness-based neighborhood. Information Sciences, 316, 180-200.

[15] Gu, W., Yin, M., \& Wang, C. (2012) Self adaptive artificial bee colony for global numerical optimization. IERI Procedia, 1, 59-65.

[16] Herrera, F., \& Lozano, M. (2003) Fuzzy adaptive genetic algorithms: design, taxonomy, and future directions. Soft Computing, 7, 545-562.

[17] Karaboga, D. (2005) An Idea Based on Honeybee Swarm for Numerical Optimization. Technical Report TR06, Erciyes University, Engineering Faculty, Computer Engineering Department.

[18] Karaboga, D., \& Akay, B. (2009) A comparative study of artificial bee colony algorithm. Applied Mathematics and Computation, 214, 108-132.

[19] Karaboga, D., \& Ozturk, C. (2011) A novel clustering approach: artificial bee colony (ABC) algorithm. Applied Soft Computing, 11(1), 652-657.

[20] Luo, J., Wang, Q., \& Xiao X. (2013) A modified artificial bee colony algorithm based on converge-onlookers approach for global optimization. Applied Mathematics and Computation, 219, 10253-10262.

[21] Pan, Q. K., Tasgetiren, M. F., Suganthan, P. N., \& Chua, T. J. (2011) A discrete artificial bee colony algorithm for the lot-streaming flow shop scheduling problem. Information Sciences, 181(12), 2455-2468.

[22] Perez, J., Valdez, F., Roeva, O., \& Castillo, O. (2016) Parameter adaptation of the bat algorithm, using type-1, interval type-2 fuzzy logic and intuitionistic fuzzy logic. Notes on Intuitionistic Fuzzy Sets, 22(2), 87-98.

[23] Pham, D. T., Ghanbarzadeh, A., Koc, E., Otri, S., Rahim, S., \& Zaidi, M. (2005) The Bees Algorithm. Technical Report, Manufacturing Engineering Centre, Cardiff University, UK.

[24] Prieto, J. A. F., \& Pérez, J. R. V. (2008) Adaptive genetic algorithm control parameter optimization to verify the network protocol performance. Proceedings of Information Processing and Management Uncertainty'08, 785-791. 
[25] Roeva, O., \& Atanassov, K. (2008) Generalized net model of a modified genetic algorithm. Issues in Intuitionistic Fuzzy Sets and Generalized Nets, 7, 93-99.

[26] Roeva, O., \& Melo-Pinto, P. (2013) Generalized net model of firefly algorithm. Proceedings of the 14th International Workshop on Generalized Nets, Burgas, 22-27.

[27] Roeva, O., \& Michalíková, A. (2014) Intuitionistic fuzzy logic control of metaheuristic algorithms' parameters via a generalized net. Notes on Intuitionistic Fuzzy Sets, 20(4), $53-58$.

[28] Roeva, O., \& Pencheva, T. (2010) Generalized net model of a multi-population genetic algorithm. Issues in Intuitionistic Fuzzy Sets and Generalized Nets, 8, 91-101.

[29] Roeva, O., Perez, J., Valdez, F., \& Castillo, O. (2016) Intercriteria analysis of bat algorithm with parameter adaptation using type-1 and interval type-2 fuzzy systems. Notes on Intuitionistic Fuzzy Sets, 22(3), 91-105.

[30] Roeva, O., Shannon, A., \& Pencheva, T. (2012) Description of simple genetic algorithm modifications using generalized nets. Proceedings of the 6th IEEE International Conference on Intelligent Systems 2012, Sofia, Bulgaria, Vol. 2, 178-183.

[31] Sombra, A., Valdez, F., Melin, P., \& Castillo, O. (2013) A new gravitational search algorithm using fuzzy logic to parameter adaptation. Proceedings of the 2013 IEEE Congress on Evolutionary Computation, 1068-1074.

[32] Valdez, F., Melin, P., \& Castillo, O. (2014) A survey on nature-inspired optimization algorithms with fuzzy logic for dynamic parameter adaptation. Expert Systems with Applications, 41(14) 6459-6466.

[33] Zhu, G., \& Wong, S. (2010) Gbest-guided artificial bee colony algorithm for numerical function optimization. Applied Mathematics and Computation, 217(7), 3166-3173.

[34] Zoteva, D., Atanassova, V., \& Roeva, O. (2018) Generalized net model of artificial bee colony optimization algorithm. IEEE Proceedings of the Advances in Neural Networks and Applications 2018, in press. 